\title{
Pathologies bucco-dentaires chez les enfants pris en charge sous anesthésie générale à l'hôpital général Idrissa Pouye de Dakar
}

\section{Mouth and tooth diseases in children treated under general anesthesia at the Idrissa Pouye general hospital in Dakar}

\author{
Djiré $\mathrm{H}^{1}$, Diakité $\mathrm{Y}^{2}$, Traoré $\mathrm{IC}^{1}$, Keita $\mathrm{DB}^{3}$, Niang $\mathrm{A}^{4}$, Kané $A S T^{5}$, Sangaré $Z^{6}$
}

\section{DOI : 10.53318/msp.v11i1.1884}

${ }^{1}$ Service d'odontostomatologie de l'hôpital de Sikasso, Sikasso, Mali 2 Service d'odontostomatologie de l'hôpital général Idrissa Pouye de Dakar, Dakar, Sénégal

${ }^{3}$ Service d'odontostomatologie de l'hôpital Fousseyni Daou de kayes, Kayes, Mali

${ }^{4}$ Service de pédodontie et de prévention du CHU-CNOS, Bamako, Mali ${ }^{5}$ Service d'odontostomatologie de l'hôpital militaire de Bamako, Bamako, Mali

${ }^{6}$ Service de cardiologie de l'hôpital mère-enfant de Kayes, Kayes, Mali

Auteur correspondant : Dr. Djiré Hamed

Service d'odontostomatologie de l'hôpital de Sikasso, Sikasso, Mali

\section{RESUME}

Introduction : La santé buccodentaire fait partie intégrante de la santé globale des enfants. Son altération peut avoir des répercussions fonctionnelles, psychologiques et sociales sur le bien-être de l'enfant. Les pathologies bucco-dentaires peuvent être source de cette altération. L'objectif de ce travail était d'évaluer les aspects épidémiologiques, cliniques et thérapeutiques des pathologies bucco-dentaires chez les enfants pris en charge sous anesthésie générale au service d'odontostomatologie de l'hôpital général Idrissa Pouye (HOGIP) de Dakar. Matériel et méthodes : II s'agissait d'une étude transversale descriptive qui s'est déroulée sur une période de 3 mois du 15 juillet 15 octobre 2019 et a concernée 25 dossiers de patients d'âge pédiatrique. Elle a eu pour cadre le service d'odontostomatologie de l'hôpital général Idrissa Pouye de Dakar. Nous avons exploité les dossiers des patients pris en charge sous anesthésie générale. Résultats : Dans notre étude, nous avons une prédominance masculine avec $56 \%$. La tranche d'âge des 6 à 10 ans représentait $48 \%$ de l'échantillon avec une moyenne de 7,8 ans $\pm 3,5$. La symptomatologie clinique était dominée par la tuméfaction ( $80 \%$ des cas). Les pathologies tumorales étaient le plus souvent rencontrées (14 cas, 56\%). Parmi ces tumeurs, la grenouillette était retrouvée dans 9 cas $(64,29 \%)$. La marsupialisation et le cerclage péri-mandibulaire ont été les interventions les plus pratiquées (respectivement $36 \%$ et $32 \%$ des cas). Conclusion : La prise en charge de ces pathologies peut se faire soit sous anesthésie locale ou soit sous anesthésie générale. Certaines situations, cliniques ou générales de l'enfant, nécessitent le recourt à l'anesthésie générale pour une meilleure prise en charge. Mots clés : Pathologies bucco-dentaires, enfants, anesthésie générale, Dakar.

\section{ABSTRACT}

Introduction: Oral health is integral to the overall health of children. Its alteration can have functional, psychological and social repercussions on the well-being of the child. Oral pathologies can be the source of this alteration. The objective of this work was to assess the epidemiological, clinical and therapeutic aspects of oral pathologies in children treated under general anesthesia at the odontostomatology department of the Idrissa Pouye General Hospital (HOGIP) in Dakar. Material and methods: This was a descriptive cross-sectional study that took place over a 3-month period from July 15 to October 15, 2019 and involved 25 files of pediatric patients. She worked in the odontostomatology department of the Idrissa Pouye general hospital in Dakar. We used the files of patients treated under general anesthesia. Results. In our study, we have a male predominance with $56 \%$. The 6 to 10 year old age group represented $48 \%$ of the sample with an average of $7.8 \pm$ 3.5 years. The clinical symptomatology was dominated by swelling ( $80 \%$ of cases). Tumor pathologies were most often encountered (14 cases, $56 \%$ ). Among these tumors, the frog was found in 9 cases $(64.29 \%)$. Marsupialization and peri-mandibular cerclage were the most performed procedures (36\% and $32 \%$ of cases, respectively). Conclusion: The management of these pathologies can be done either under local anesthesia or under general anesthesia. Certain situations, clinical or general in the child, require the use of general anesthesia for better management.

Keywords: Oral pathologies, children, general anesthesia, Dakar.

\section{INTRODUCTION}

La santé buccodentaire fait partie intégrante de la santé globale des enfants. Son altération peut avoir des répercussions fonctionnelles, psychologiques et sociales sur le bien-être de l'enfant [1]. Les pathologies buccodentaires peuvent être source de cette altération [2]. Celles-ci peuvent être groupées en pathologies infectieuses, traumatiques, tumorales [3,4]. Parmi les pathologies infectieuses, la carie dentaire et ses complications est la principale maladie infantile chronique qui cause un problème de santé publique [5]. Quant- aux pathologies traumatiques, elles sont très fréquentes pendant l'enfance et peuvent avoir des conséquences négatives sur la croissance des bases osseuses maxillomandibulaires [6].

La prévalence des traumatismes maxillo-faciaux est estimée à $20 \%$ chez l'enfant en Afrique [7]. Près d'un tiers de la population mondiale serait concernée par des 
tumeurs buccales, avec une nette prédominance des lésions bénignes [8]. De ce fait, l'utilisation de l'anesthésie générale dans la population pédiatrique devient cruciale. Ses indications sont liées soit à l'état général du patient, soit à l'intervention et soit à une éventuelle contreindication de l'anesthésie locale [9]. Toutefois, malgré toutes les avancées en anesthésie-réanimation, la décision d'intervenir sous anesthésie générale chez l'enfant doit être réfléchie [10]. L'objectif de notre étude était d'évaluer les aspects épidémiologiques, cliniques et thérapeutiques des pathologies bucco-dentaires chez les enfants pris en charge sous anesthésie générale au service d'odontostomatologie de l'hôpital général Idrissa Pouye (HOGIP) de Dakar.

\section{MATERIEL ET METHODES}

II s'agissait d'une étude transversale descriptive à partir de dossiers d'enfants pris en charge sous anesthésie générale. L'étude s'est déroulée sur une période de trois mois, allant du 15 juillet au 15 octobre 2019.

Les dossiers sélectionnés étaient ceux des patients d'âge pédiatrique suivis au service d'odontostomatologie de l'hôpital général Idrissa Pouye de Dakar. Etaient inclus dans l'étude, tout patient âgé de 0 à 15 ans pris en charge au bloc opératoire sous anesthésie générale. N'étaient pas inclus, tout patient âgé de 0 à 15 ans dont le dossier médical était mal rempli. Nous avons effectué un recrutement exhaustif. Durant cette période, vingt-neuf (29) ont été recueillis au total. Quatre dossiers n'ont pas été inclus parce qu'ils étaient mal remplis. Vingt-cinq (25) dossiers ont été retenus pour l'étude.

Les variables étudiées étaient les données sociodémographiques (sexe, âge), les données cliniques (symptomatologie, pathologies rencontrées), les données paracliniques (radiographie panoramique, tomodensitométrie), les données thérapeutiques (traitement médicamenteux, type d'intervention réalisée), les données portant sur l'évolution post opératoire.

Une fiche d'enquête a été élaborée pour cette étude. Cette fiche d'enquête a reçu l'approbation des responsables de la structure. Elle ne comprend ni le nom, ni le prénom du patient, ceci dans le souci de respecter la confidentialité et la protection des données personnelles du patient.

Les données ont été recueillies à l'aide des dossiers de patients et d'une fiche d'enquête établie pour la circonstance. Elles ont ensuite été analysées à l'aide du logiciel Epi Info version 7.2.3.1. Des analyses unies variées ont été effectuées.

\section{RESULTATS}

\section{- Données sociodémographiques}

Le sexe masculin était le plus représenté avec $56 \%$ des cas. Le sex-ratio $(H / F)$ était de 1,3. La tranche d'âge la plus représentée était de 6 à 10 ans soit $48 \%$ des cas. L'âge moyen des patients était de 7,8 ans $\pm 3,5$ avec des extrêmes de 2 et 15 ans et un écart type (tableau I).

\section{- Données cliniques}

La symptomatologie la plus rencontré était la tuméfaction dans $80 \%$ des cas, le trismus et l'asymétrie faciale dans $76 \%$ chacun, la douleur dans $40 \%$ et la mobilité maxillaire dans $36 \%$ (figure 1 ).

Les tumeurs et les fractures mandibulaires étaient les plus rencontrées avec respectivement $56 \%$ et $32 \%$ des cas (figure 2).

Parmi les tumeurs, le kyste mucoïde du plancher buccal (grenouillette) était le plus retrouvé avec $64,29 \%$ des cas (tableau II).

- Données paracliniques

La tomodensitométrie (TDM) a été la plus réalisée avec $60 \%$ des cas, suivi de l'orthopantomagramme (OPG) dans $40 \%$ des cas.

\section{- Données thérapeutiques}

La marsupialisation et le cerclage péri-mandibulaire étaient plus retrouvés avec respectivement $36 \%$ et $32 \%$ des cas (figure 3).

- Données portant sur l'évolution post opératoire Les suites opératoires étaient bonnes dans $96 \%$ des cas. Une complication infectieuse a été retrouvée après un cerclage péri-mandibulaire.

\section{DISCUSSION}

La tranche d'âge la plus représentée dans la présente étude était 6 à 10 ans soit $48 \%$ des cas. Des études réalisées en Angleterre en 2014 par Raja et al. et en 2016 par Tahmassebi et al. ont rapporté des tranches d'âge similaires [11, 12]. Par contre Sanabria et al. à Bogota (Colombie) en 2018 et Kakaounaki et al. en 2011 ont trouvé des tranches d'âge supérieures dans leur série, soit $10-14$ ans $(31,05 \%)$ [2, 13]. Cette différence pourrait s'expliquer par la taille de l'échantillon.

Dans notre étude, les tumeurs et les fractures de la mandibule étaient les plus retrouvées avec respectivement $56 \%$ et $32 \%$ des cas. Au Nigéria en 2018 , Ibikunle et al. ont rapportée une prédominance des tumeurs bénignes avec $30,4 \%$ des cas [14]. Par contre, en République Tchèque en 2019, Ivancakova avait trouvé $81,22 \%$ cas de carie dentaire et ses complications qui ont été pris en charge sous anesthésie générale [15]. Ces résultats sont différents de ceux de notre étude où seuls 2 enfants porteurs de caries dentaires ont été traités sous anesthésie générale car ils présentaient une déficience mentale qui empêchait toute coopération pour les soins. Parmi les tumeurs, le kyste mucoïde du plancher buccal (grenouillette) était retrouvé dans $64,29 \%$ des cas. Des taux plus faible ont été rapportés par Prosdócimo et al. en 2018 au Brésil avec 22\% de kyste mucoïde du plancher [16]. Outre les aspects cliniques, des examens complémentaires ont été également demandés.

La tomodensitomètrie était l'examen radiologique le plus demandée suivie de l'orthopantomogramme avec respectivement $60 \%$ et $40 \%$ des cas. Ces résultats sont similaires à ceux retrouvés dans une étude réalisée en Baltimore en 2015 par Ghasemzadeh et al., où la tomodensitométrie était réalisée chez tous les patients présentant une fracture mandibulaire [17]. En effet, dans 
la population de patients pédiatriques, il est judicieux de compléter l'orthopantomogramme par une tomodensitométrie.

La marsupialisation et le cerclage péri-mandibulaire étaient plus retrouvés avec respectivement $36 \%, 32 \%$ des cas. La prédominance de la marsupialisation est liée au nombre plus élevé d'enfants ayant présenté une grenouillette volumineuse dont la prise en charge se fait le plus souvent par marsupialisation sous anesthésie générale. Pour le cerclage péri mandibulaire, sa prédominance dans la prise en charge des fractures de la mandibule s'explique d'une part par la non disponibilité des mini plaques dans le service, mais également par le souci de préserver les germes des dents permanentes pouvant être traumatisés par les vises des mini plaques. Dans la prise en charge des fractures mandibulaires, Dia et al. au Sénégal en 2009 [18] avait réalisé un cerclage péri-mandibulaire dans $13,59 \%$ des cas largement inférieur aux valeurs rapportées dans la présente étude. Cela pourrait s'expliquer par le fait que seuls les enfants étaient inclus dans la présente étude mais également dans le souci de préserver les germes des dents permanentes pouvant être lésées par les vises des mini plaques.

En postopératoire les suites étaient bonnes dans $96 \%$ des cas avec une seule complication infectieuse après la prise en charge d'une fracture mandibulaire. Cette complication pourrait s'expliquer par les soucis rencontrés au cours de l'intervention ayant amené le praticien à modifier le protocole opératoire.

\section{CONCLUSION}

Les pathologies bucco-dentaires chez les enfants sont diverses et variées. Elles peuvent poser un véritable défi de diagnostic et de prise en charge.

La prise en charge de certaines d'entre elles est possible sous anesthésie locale. Mais d'autres exigent l'anesthésie générale selon la situation clinique et/ou l'état général de l'enfant.

Après l'analyse des résultats, cette étude nous a permis de savoir que l'utilisation de l'anesthésie générale est très peu documentée dans les pays africains. Une meilleure collaboration avec les anesthésistes réanimateurs pourra permettre aux chirurgiens-dentistes (surtout ceux spécialisés en odontologie pédiatrique) d'effectuer les soins dentaires chez les enfants sous anesthésie générale dans notre contexte.

\section{REFERENCES}

1. Société canadienne de pédriatrie. Les soins buccodentaires des enfants - un appel à l'action. Paediatr Child Health. 2013;18(1):44-50.

2. Sanabria EAP, Patricia C. Frequency of oral and maxillofacial pathologies in patients from 0 to 18 years in the fundación hospital de la Misericordia Bogotá (Colombia), during the Period 2006-2014. Universitas Médica. 2018;59(4):1-14.
3. Anyanechi CE, Saheeb BD. Audit of demographic trends of oral and maxillofacial surgery cases at a Nigerian University Hospital. College of Med. 2011;16:18-27.

4. Omitola DG, Osagbemiro B, Akadiri OA. Spectrum of disease and reference model at the oral diagnosis clinic of a tertiary dental center. Nig Dent J. 2011;19:66-70.

5. Lorca B. Quelles sont les connaissances actuelles des parents sur la prévention buccodentaire de leur(s) enfant? Thèse Med. Toulouse; 2013, №1080, 51p.

6. Brizon JC. Anatomie : Vaisseaux du cou. Paris Maloines. 1999, 5199-5367.

7. Glendor U, Andersson L. Public health aspects of oral diseases and disorders: Dental trauma. In Pine C, Harris $\mathrm{R}$, editors. Community oral health, London, Quintessence Publishing, 2007

8. Khatib KE et al. Tumeurs bénignes de la cavité buccale : étude rétrospective de 209 cas. Med Buc Chir Buc. 2011;17:115-119.

9. Musilli J. Soins dentaires sous anesthésie générale chez l'enfant : réalisation d'une plaquette d'information à destination des parents. Thèse Chir Dent. Lille ; 2017, No1021, 85p.

10. Bandon D, Prevost J, Brun-Croese N. L'anesthésie générale en odontologie pédiatrique. J Odontstomatol Pediatr. 2003;10(2):67-76.

11. Raja A, Daly A, Harper R, Senghore N, White1 D and Ravaghi V. Characteristics of children undergoing dental extractions under general anaesthesia in Wolverhampton: 2007-2012. Br Dent J. 2016;220(8):40711.

12. Tahmassebi JF, Achol LT, Fayle SA. Analysis of dental care of children receiving comprehensive care under general anesthesia at a university hospital in England. Eur Arch Paediatr Dent. 2014;15(5):353-60.

13. Kakaounaki E, Tahmassebi JF, Fayle SA. Repeat general anaesthesia, a 6-year follow up. Int J Paediatr Dent. 2011;21(2):126-31.

14. Ibikunle AA, Taiwo AO, Braimah RO. A 5-year audit of major maxillofacial surgeries at Usmanu Danfodiyo university teaching hospital, Nigeria. BMC Health Services Research. 2018;18:4.

15. Ivancakova RK, Suchanek J, Kovacsova F, Cermakova E, Merglova V. The Analysis of Dental Treatment under General Anaesthesia in Medically Compromised and Healthy Children. Int J Environ Res Public Health. 2019; 16:25-28.

16. Prosdócimo ML, Agostini M, Romañach MJ, Andrade $B A B$. A retrospective analysis of oral and maxillofacial pathology in a pediatric population from Rio de Janeiro-Brazil over a 75-year period. Med Oral Patol Oral Chir Buc. 2018;23(5):511-7.

17. Ghasemzadeh A et al. Treatment of Pediatric Condylar Fractures: à 20-year experience. Plast Reconstr Surg. 2015;136(6):1279-1288.

18. Tine SD. Fractures de la mandibule en pratique odontologique : à propos de 103 cas. Med Buc, Chir Buc. 2009;3(15):137. 
LISTE DES TABLEAUX ET FIGURES

Tableau I : Répartition de la population selon la tranche d'âge.

\begin{tabular}{lcc}
\hline Tranche d'âge (ans) & Effectif & $\%$ \\
\hline $0-5$ & 3 & 12 \\
\hline $6-10$ & 12 & 48 \\
\hline $11-15$ & 10 & 40 \\
\hline Total & $\mathbf{2 5}$ & $\mathbf{1 0 0}$ \\
\hline
\end{tabular}

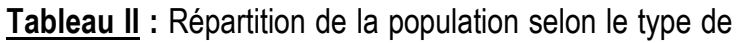
tumeur.

\begin{tabular}{lcc}
\hline Type de tumeur & Effectif & $\%$ \\
\hline $\begin{array}{l}\text { Kyste mucoïde du } \\
\text { plancher buccal }\end{array}$ & 9 & 64,29 \\
\hline Kystes folliculaire & 4 & 28,57 \\
\hline Epulis & 1 & 7,14 \\
\hline Total & $\mathbf{1 4}$ & $\mathbf{1 0 0}$ \\
\hline
\end{tabular}

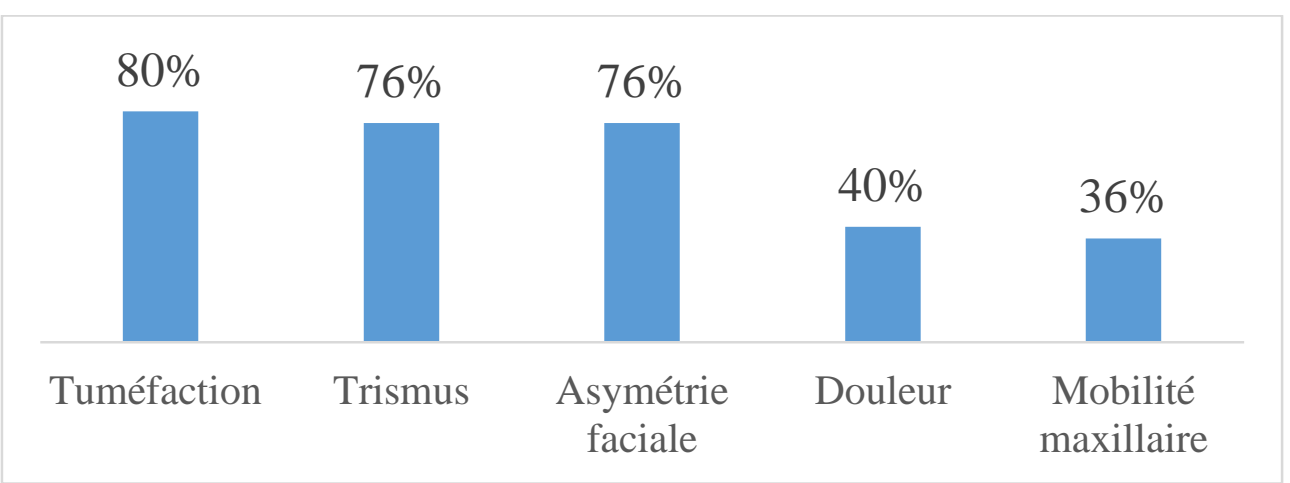

Figure 1: Répartition de la population selon la symptomatologie clinique.

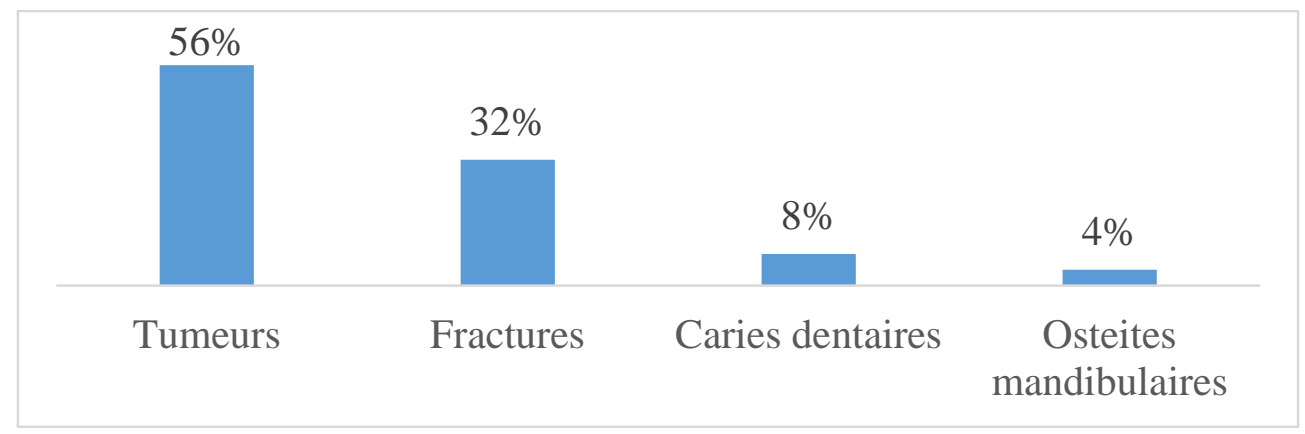

Figure 2: Répartition de la population selon les pathologies rencontrées.

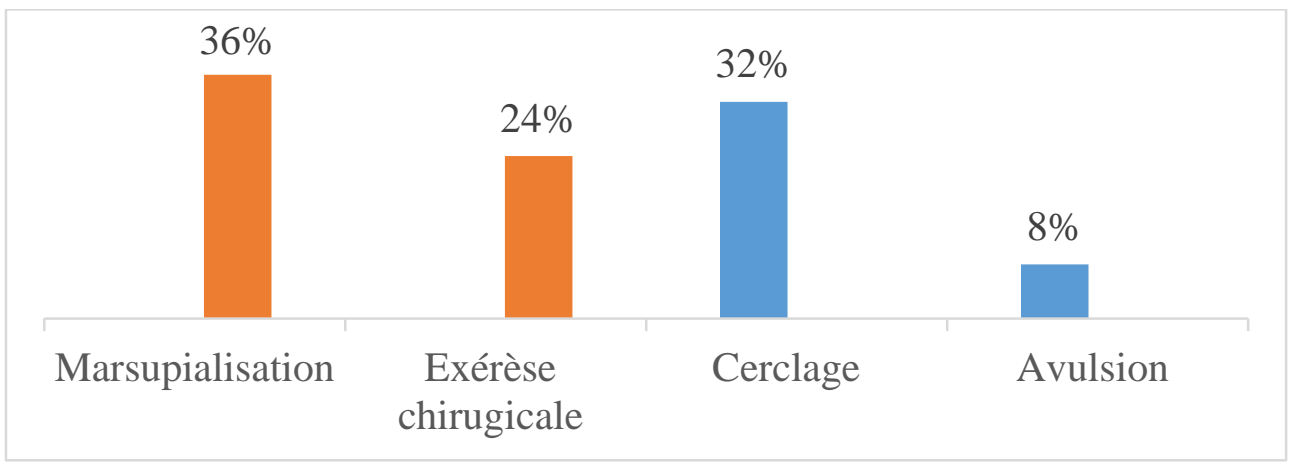

Figure 3: Répartition de la population selon le type d'intervention. 